\title{
The Effect of Inflammatory Cytokines in Alcoholic Liver Disease
}

\author{
Hideto Kawaratani, Tatsuhiro Tsujimoto, Akitoshi Douhara, Hiroaki Takaya, Kei Moriya, \\ Tadashi Namisaki, Ryuichi Noguchi, Hitoshi Yoshiji, Masao Fujimoto, and Hiroshi Fukui
}

Third Department of Internal Medicine, Nara Medical University, 840 Shijo-cho, Kashihara, Nara 634-8522, Japan

Correspondence should be addressed to Hideto Kawaratani; kawara@naramed-u.ac.jp

Received 1 September 2013; Accepted 12 November 2013

Academic Editor: Borna Relja

Copyright (C) 2013 Hideto Kawaratani et al. This is an open access article distributed under the Creative Commons Attribution License, which permits unrestricted use, distribution, and reproduction in any medium, provided the original work is properly cited.

\begin{abstract}
Alcohol is the most common cause of liver disease in the world. Chronic alcohol consumption leads to hepatocellular injury and liver inflammation. Inflammatory cytokines, such as TNF- $\alpha$ and IFN- $\gamma$, induce liver injury in the rat model of alcoholic liver disease (ALD). Hepatoprotective cytokines, such as IL-6, and anti-inflammatory cytokines, such as IL-10, are also associated with ALD. IL6 improves ALD via activation of the signal transducer and activator of transcription 3 (STAT3) and the subsequent induction of a variety of hepatoprotective genes in hepatocytes. IL-10 inhibits alcoholic liver inflammation via activation of STAT3 in Kupffer cells and the subsequent inhibition of liver inflammation. Alcohol consumption promotes liver inflammation by increasing translocation of gut-derived endotoxins to the portal circulation and activating Kupffer cells through the LPS/Toll-like receptor (TLR) 4 pathways. Oxidative stress and microflora products are also associated with ALD. Interactions between pro- and anti-inflammatory cytokines and other cytokines and chemokines are likely to play important roles in the development of ALD. The present study aims to conduct a systemic review of ALD from the aspect of inflammation.
\end{abstract}

\section{Introduction}

Alcohol-related liver disease is a major cause of morbidity and mortality worldwide. Chronic alcohol consumption leads to hepatocellular injury, fat accumulation, and liver inflammation and sometimes leads to liver cirrhosis or hepatocellular carcinoma (Figure 1). The pathogenesis of alcoholic liver disease (ALD) is a consequence of chronic alcohol consumption. The clinical syndrome of ALD carries a particularly poor prognosis, such as liver cirrhosis [1] or hepatocellular carcinoma [2]. The pathogenesis of ALD is uncertain, but the relevant factors include metabolism of alcohol to toxic products, oxidative stress, acetaldehyde adducts, abnormal methionine metabolism, malnutrition, the activation of endotoxin, and impaired hepatic regeneration (Figure 2) [3]. Kupffer cells, the resident macrophages in the liver, play the role of an innate immune system; they produce various cytokines and are known to be involved in the pathogenesis of liver diseases [4]. The inflammatory cytokine, tumor necrosis factor-alfa (TNF- $\alpha$ ), is involved in acute alcoholic liver injury [5]. Moreover, it is also well known that chronic alcohol consumption increases TNF- $\alpha$ production and leads to liver injury [6].
The consumption of alcohol leads to an augmented permeability of the intestinal membrane, which increases the portal concentration of blood endotoxin (lipopolysaccharide; LPS) [7]. This causes the Kupffer cells to be activated and exhibit enhanced sensitivity to LPS-stimulated inflammatory cytokine production [8]. Chronic alcohol consumption leads to injury of the hepatocytes by TNF- $\alpha$, with consequent apoptosis and phagocytosis by the Kupffer cells. The Kupffer cells are activated by phagocytosing the apoptotic cells and their inflammatory cytokine production is increased [9]. Alcohol consumption promotes liver inflammation by increasing the translocation of gut-derived endotoxins to the portal circulation and activating Kupffer cells through the LPS/Tolllike receptor (TLR) 4 pathways. ALD is associated with imbalanced immune responses and increased production of proinflammatory cytokines and chemokines [10, 11]. Various cytokines are associated with ALD, including hepatoprotective cytokines, such as lnterleukin-6 (IL-6), and anti-inflammatory cytokines, such as IL-10 [12]. These two cytokines are produced by ethanol-induced LPS-stimulated Kupffer cells and can attenuate alcohol-induced liver injury. Inflammasome and several chemokines also contribute to ALD. 


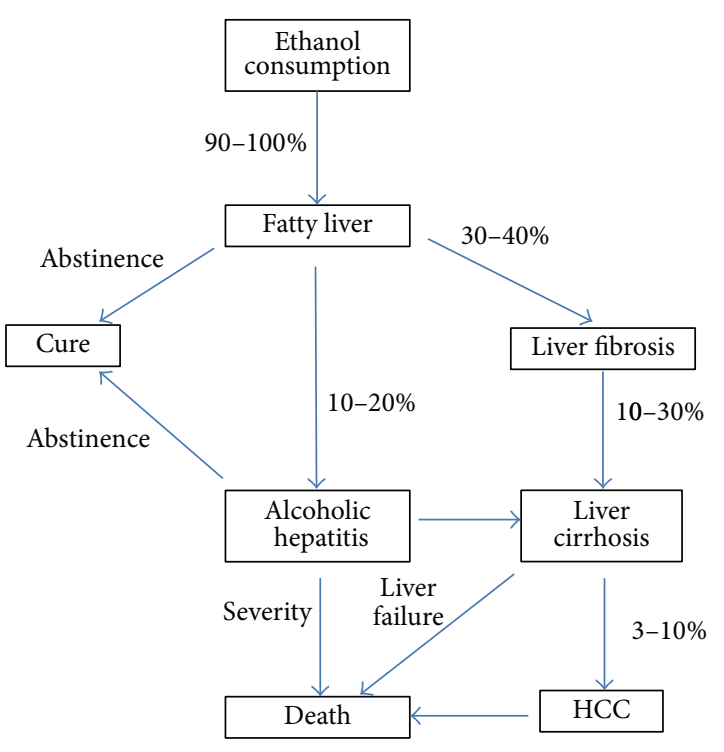

FIgUre 1: The natural history of alcoholic liver disease. Chronic ethanol consumption leads to fatty liver for more than $90 \%$. But only up to $40 \%$ of this population develops more severe forms of alcoholic liver disease (ALD), including fibrosis and alcoholic hepatitis. Continuous ethanol consumption finally leads to liver cirrhosis or hepatocellular carcinoma and leads to death.

Thus, the present paper aims to conduct a systemic review of ALD from the aspect of inflammation.

\section{Metabolism of Alcohol}

When alcohol is consumed, it passes from the stomach and intestines into the blood, a process referred to as absorption. Alcohol then enters the liver through the portal vein. We show the pathway of alcohol metabolism in Figure 3. In the liver, alcohol dehydrogenase $(\mathrm{ADH})$, the key enzyme in alcohol metabolism, mediates the conversion of alcohol to acetaldehyde [13]. Acetaldehyde is rapidly converted to acetate by acetaldehyde dehydrogenase (ALDH) and is eventually metabolized in the muscle to carbon dioxide and water. There is another pathway independent of $\mathrm{ADH}$, which is called the microsomal ethanol oxidizing system (MEOS) [14]. Alcohol is metabolized in the liver by the enzyme cytochrome P450 2E1 (CYP2E1). CYP2E1 is mainly expressed in the liver, with hepatocytes showing the highest expression, but it is also located in other organs, such as the brain and intestine. CYP2E1 is mainly located within the endoplasmic reticulum (ER) although it is also expressed in the mitochondria [15] and is increased after chronic alcohol consumption [16] and an increase in acetaldehyde. Acetaldehyde has a stronger toxicity than ethanol and leads to liver injury. Most of the alcohol consumed is metabolized in the liver, but the small quantity that remains is not metabolized and is excreted in the breath and urine.

2.1. TNF- $\alpha$. TNF- $\alpha$ is a cytokine involved in systemic inflammation and is a member of a cytokine family that stimulates

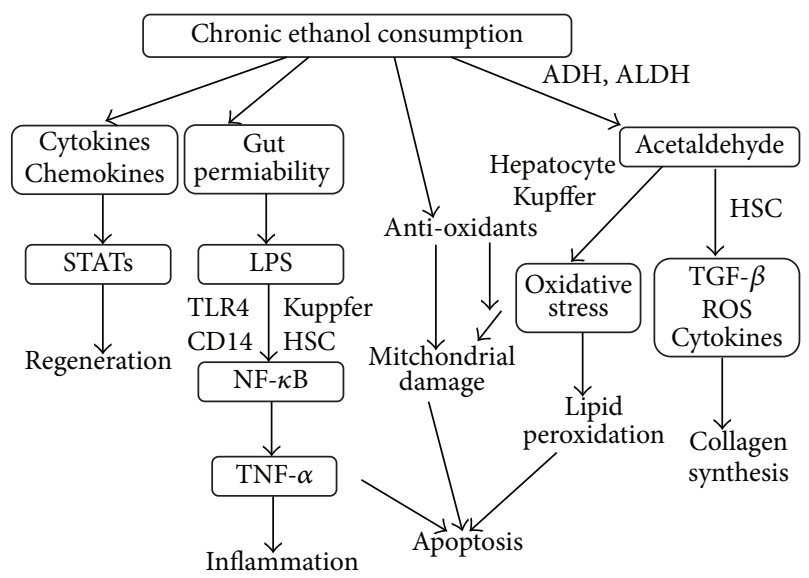

FIGURE 2: The pathogenic mechanisms of alcoholic liver disease. Chronic ethanol consumption promotes the translocation of LPS from the intestine to the portal vein, where it binds to the lipopolysaccharide-binding protein (LBP). STATs induces liver regeneration. Ethanol consumption alters the intracellular balance of antioxidants with subsequent decrease in the release of mitochondrial damage, leading to hepatic apoptosis. Hepatocytes and activated Kupffer cells are suggested to be the sources of oxidative stress, which are responsible for lipid peroxidation and further apoptotic damage. Activation of hepatic stellate cells also contributes to the production of TGF- $\beta$, ROS, and cytokines, leading to liver fibrosis.

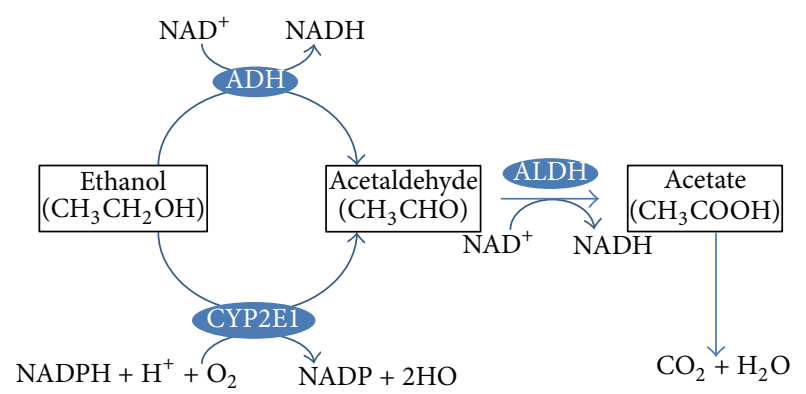

FIGURE 3: The pathway of ethanol metabolism. Ethanol is metabolized into acetaldehyde by alcohol dehydrogenase $(\mathrm{ADH})$ and the microsomal enzyme cytochrome P450 2E1 (CYP2E1). The $\mathrm{ADH}$ enzyme reaction is the main ethanol metabolic pathway involving an intermediate carrier of electrons, namely, nicotinamide adenine dinucleotide $\left(\mathrm{NAD}^{+}\right)$. Acetaldehyde is rapidly metabolized by aldehyde dehydrogenase (ALDH) in the mitochondria to acetate and NADH. And acetate is eventually metabolized in the muscle to carbon dioxide and water.

acute inflammation. TNF- $\alpha$ is produced by various types of cells in the body. In the liver, TNF- $\alpha$ is mainly produced by Kupffer cells, and TNF- $\alpha$ is also an important mediator in various physiological processes, such as inflammation, cell proliferation, and apoptosis [10]. The role of TNF- $\alpha$ as a critical inflammatory cytokine in the progression of ALD is now well known [5]. However, the mechanism of alcohol enhancement of TNF- $\alpha$ has not been clarified yet. Kupffer cells secrete inflammatory cytokines [4] and reactive oxygen species (ROS) [17], which activate cells such as hepatocytes, hepatic 
stellate cells, and endothelial cells [18]. In alcoholic hepatitis $(\mathrm{AH})$, inflammatory cytokines, such as TNF- $\alpha$ or IL-6, induce liver injury [19]. After chronic alcohol consumption, Kupffer cells exhibit enhanced sensitivity to LPS-stimulated TNF- $\alpha$ production [20]. Elevated serum levels of TNF- $\alpha$ inducible cytokines or chemokines, including IL-6, IL-8, and IL-18, have also been reported in patients with AH [21]. Serum TNF- $\alpha$ is increased in patients with ALD and correlates with mortality. Administration of excessive ethanol to TNF$\alpha$ knockout mice does not cause liver injury. Thus TNF- $\alpha$ is thought to be the main cytokine of inflammation. Furthermore, increased serum levels of TNF- $\alpha$ have also been noticed in rat models of nonalcoholic steatohepatitis (NASH) [22] and in patients with NASH [23]. TNF- $\alpha$ is associated with the development of liver injury in both ALD and NASH.

Recently, it has become known that platelet aggregation activity is associated with ALD. The platelet adhesive protein, von Willebrand factor (VWF), and its cleavage protease, ADAMTS13, have been gaining attention. In previous studies, our group showed that plasma ADAMTS13 activity decreased in ALD or severe $\mathrm{AH}$ and was inversely proportional to TNF- $\alpha$ [24-26]. Treatment with pentoxifylline, an inhibitor of TNF- $\alpha$ synthesis, improved the survival of patients with severe AH [27]. Anti-TNF- $\alpha$ antibodies prevented inflammation and necrosis in the rat model of alcohol feeding [6]. Anti-TNF- $\alpha$ antibody, infliximab, is also effective in severe $\mathrm{AH}$ patients [28]. Multiple cytokine modulator, Y-40138, is known to inhibit the production of inflammatory cytokines, such as TNF- $\alpha$ or IL- 6 , and to enhance the production of antiinflammatory cytokines, such as IL-10. Our results showed that Y-40138 reduced the inflammatory cytokines in ALD [29]. These results suggest that TNF- $\alpha$ plays an important role in the progression of ALD.

2.2. IL-6. The role of IL-6 in ALD is complex and not well understood. It appears to have some beneficial effects on the liver. IL-6 may protect against hepatocyte apoptosis and participate in mitochondrial DNA repair after alcoholic liver injury [30, 31]. IL-6 may promote human Th17 differentiation and IL-17 production, therefore contributing to ethanolinduced liver inflammation. IL-6 is also released along with IL-10, TNF- $\alpha$, and other cytokines by Kupffer cells after alcohol consumption. IL- 6 and IL-10 are two cytokines that play roles in reducing alcoholic liver injury and inflammation through activation of the signal transducer and activator of transcription (STAT3) [12]. Elevated IL-6 is found in chronic alcohol-fed animals and in alcoholics, with or without liver disease [32]. On the other hand, IL-6 knockout mice fed chronic alcohol showed increased liver fat accumulation, lipid peroxidation, mitochondrial DNA damage, and sensitization of hepatocytes to TNF- $\alpha$ induced apoptosis, which was prevented by the administration of recombinant IL- $6[31,33$, 34]. Furthermore, blocking of IL-6 signalling in mice reduced the infiltration of neutrophils and mononuclear cells and inflammation [35]. These findings suggest that IL-6 has a protective effect at the early phase of ALD.
2.3. IL-10. IL-10 is an anti-inflammatory cytokine that controls the endogenous production of TNF- $\alpha$ during endotoxemia and reduces LPS stimulation when added exogenously [36]. IL-10 is produced by macrophages, lymphocytes, and Kupffer cells, and the liver is considered to be the main source of IL-10 production [37]. IL-10 decreases the production of proinflammatory cytokines, such as TNF- $\alpha$, IL-1 $\beta$, and IL- 6 , from activated macrophages or monocytes. IL-10 also possesses a hepatic protective effect on proliferation and fibrosis [38]. In the liver, Kupffer cells are the main producers of IL10. Kupffer cells produce IL-10 in response to LPS stimulation and downregulate the release of TNF- $\alpha$ and IL-6. Endotoxin administration is an extensively studied model of IL-10 induction from monocytes and macrophages [39]. Human monocytes activated by LPS are able to produce a high level of IL-10 in a dose-dependent manner [40]. The activated monocytes inhibit production of proinflammatory cytokines, such as TNF- $\alpha$, IL- 6 and IL-1 $\beta$. Moreover, proinflammatory cytokine levels were also increased by LPS treatment in IL-10 knockout mice [41]. Alcohol-fed IL-10 knockout mice have increased hepatic and systemic inflammatory conditions, and LPS enhanced alcohol-induced liver injury compared with wild-type mice [42]. On the other hand, IL-10 knockout mice have a reduced fatty liver and lower serum AST and ALT levels after ethanol feeding compared with wild-type mice [42]. This may be because IL-10 knockout mice have elevated levels of IL-6, and STAT3 activation in the liver, which lead to steatosis and hepatocellular damage. At the early stage of ALD, inflammation involving IL-6/STAT3 has a protective effect against alcoholic steatosis and liver injury. These findings suggest that IL-10 as well as IL-6 plays a protective role in the progression of ALD.

2.4. Other Interleukins. Nuclear regulatory factor kappa B $(\mathrm{NF}-\kappa \mathrm{B})$ is a protein complex that controls the transcription of DNA and a central regulator of cellular stress in all cell types in the liver. $\mathrm{NF}-\kappa \mathrm{B}$ plays a key role in regulating the immune response to infection and in both acute and chronic inflammation. Activation of NF- $\kappa \mathrm{B}$ in rats can induce the expression of IL-1 $\beta$, which increases the expression of proinflammatory molecules $[43,44]$. IL- $1 \beta$ and IL- 6 were found to be essential for the induction of Th17 lymphocyte differentiation from human naive CD4+ T cells [45]. Furthermore, LPSstimulated human monocytes induced Th17 polarization of naive $\mathrm{CD} 4^{+} \mathrm{T}$ cells in an IL-1 $\beta$ signalling-dependent manner. IL-8 is a critical proinflammatory cytokine involved in many steps of neutrophil mobilization, from bone marrow to tissue infiltration or activation. IL- 8 is induced by TNF- $\alpha$ and by ligands for TLRs via the activation of NF- $\kappa$ B. Serum IL- 8 is highly elevated in patients with $\mathrm{AH}$ and is linked to neutrophil infiltration. In contrast, IL- 8 is only moderately elevated in alcoholic cirrhosis patients or alcoholics. IL-17 plays a key role in enhancing the host immune response against microorganisms as well as in autoimmune diseases [46]. IL17 stimulates multiple types of nonparenchymal hepatic cells to produce proinflammatory cytokines and chemokines [47] with a relatively weak TNF- $\alpha$-like function. Furthermore, IL17 can act with other cytokines to activate NF- $\kappa$ B and induce 
IL-8. Recently it was shown that patients with ALD had higher IL-17 plasma levels compared with healthy subjects [48]. The functions of Th17 cells are also mediated via the production of IL-22. IL-22 is a member of the IL-10 family of cytokines and plays an important role in promoting hepatocyte survival and proliferation [49]. IL-22 administration to alcohol-fed mice also prevented liver steatosis and liver injury through the activation of hepatic STAT3 [50].

IL- $1 \beta$ is also a potent proinflammatory cytokine [51]. In both animal model and patient with ALD, the levels of proIL- $1 \beta$ are significantly increased in the liver and in the serum $[52,53]$. IL- $1 \beta$ is produced as inactive pro-IL- $1 \beta$ in response to inflammatory stimuli, including both microbial products and endogenous danger-associated molecules. IL- $1 \beta$ gene expression and synthesis of pro-IL- $1 \beta$ occur after activation of pattern recognition receptors (PRRs). IL- $1 \beta$ acts in an autocrine or paracrine manner via the type I IL-1 receptor (IL-1R1). Activation of IL-1R1 is inhibited by its binding to the IL1 receptor antagonist (IL-1Ra). Treatment with IL-1Ra significantly improves symptoms in patients with rheumatoid arthritis [54], or autoinflammatory syndromes.

\section{Toll-Like Receptors}

Toll-like receptors (TLRs), a family of PRRs, are transmembrane proteins originally identified in mammals on the basis of their homology with Toll, a Drosophila receptor that contributes to the production of antimicrobial peptides that act against microorganism invasion in the fly. 11 TLRs have been identified in humans and 13 in the mouse [55]. TLRs recognize pathogen-derived molecules, such as structural components unique to bacteria, virus, and fungi, and activate inflammatory cytokines and type I interferon (IFN) production. TLRs are expressed on the surface of immune cells, such as macrophages, dendritic cells, and nonimmune cells, including epithelial cells. Expression of TLR1, 2, 6, 7, and 8 was elevated in chronic ethanol-feeding model. The treatment with ethanol resulted in sensitization to liver inflammation and damage by TLR1, 2, 4, 6, 7, 8, and 9 ligands due to increased expression of TNF- $\alpha$ [56]. However, deficiency in TLR2 had no protective effect in a chronic ethanolfeeding mouse model [57]. TLRs play important roles in the pathophysiology of a variety of liver diseases [58], which may attribute to wide expression of TLRs on hepatocytes [59]. TLR4 is a functional receptor expressed on the surface of macrophages and various other types of cells that transmit endotoxin signals. Cluster of differentiation 14 (CD14) is a protein that is a component of the innate immune system. CD14 binds to LPS, thereby subsequently presenting it to TLR4 and MD-2, which activate the intracellular signalling pathway via myeloid differentiation factor 88 (MyD 88), resulting in NF- $\kappa \mathrm{B}$ activation [60]. Both MyD88 and TRIF (MyD88 independent) signalling are associated with TLR3 and TLR4 (Figure 4). Recruitment of the TRIF adapter activates phosphorylation of interferon regulatory factor 3 (IRF3) that results in type I IFN production [61]. It is known that mice deficient in IRF3 or TLR4 expression are protected from alcohol-induced liver inflammation and hepatocyte injury

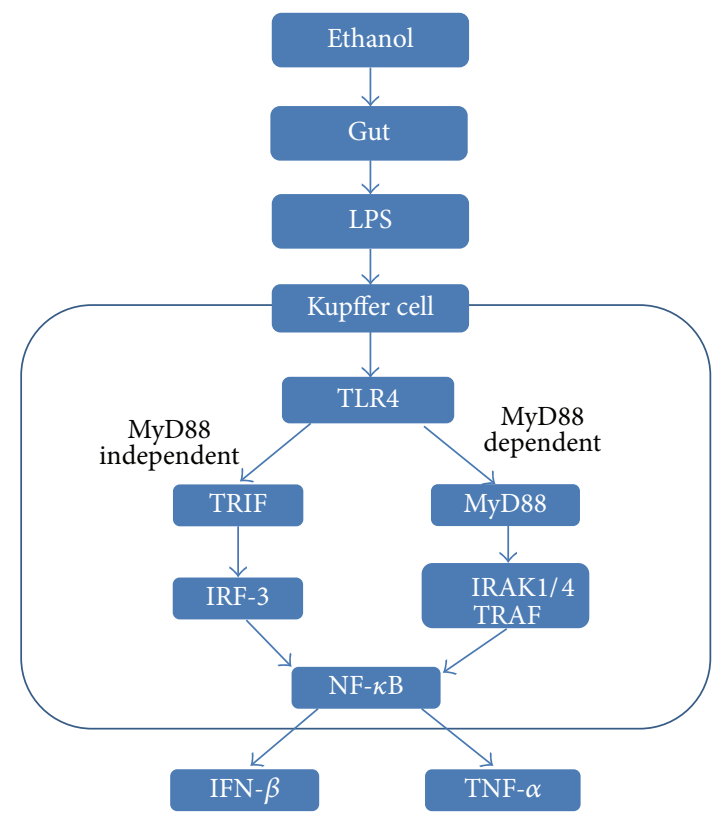

FIgURE 4: Toll-like receptor 4 signaling pathway in alcohol consumption. Ethanol promotes the translocation of lipopolysaccharide from the gastrointestinal lumen to the portal vein, where it binds to the lipopolysaccharide-binding protein. In Kupffer cells, lipopolysaccharide binds to CD14, which combines with TLR4 activating multiple cytokine genes. TLR4 are activated by MyD88 dependent or independent manner, leading to secretion of TNF- $\alpha$ or IFN- $\beta$.

[62]. The LPS/TLR4 signalling pathway consists of activation of transcription factors, such as NF- $\kappa \mathrm{B}$, which induces proinflammatory cytokine expression in the Kupffer cell. In the liver, TLR4 is expressed not only on innate immune cells such as Kupffer cells, but also on hepatocytes, hepatic stellate cells, sinusoidal endothelial cells, and biliary epithelial cells. Blockade of TLR4 or CD14 reduces liver pathology and inflammation in a mouse model of alcoholic liver injury [63, 64], which indicates the importance of the TLR4 signalling pathway. LPS recognition by TLR4 expressed on hepatic stellate cells and sinusoidal epithelial cells also contributes to the progression of ALD [65]. Alcohol stimulates Kupffer cells and monocytes to produce increased TNF- $\alpha$ in response to endotoxin [66]. In previous studies, our group showed that endotoxemia plays an important role in the initiation and aggravation of ALD through the enhancement of proinflammatory cytokines, including IL-6, IL-8, and TNF- $\alpha[67,68]$. Hepatic expression of TLR1, 2, 4, 6, 7, 8, and 9 mRNA was increased in the mouse model of chronic alcohol feeding [69]. Alcohol feeding also resulted in sensitization to liver damage and inflammation because administration of TLR1, $2,4,6,7,8$, and 9 ligands resulted in increased expression of TNF- $\alpha$ mRNA [69]. Acute alcohol exposure inhibited TLR4 signalling in macrophages after alcohol treatment in mice leading to decreased LPS-induced TNF- $\alpha$ production [70]. In ALD, TLR3 activation in HSCs and Kupffer cells plays an antagonistic role against the TLR4-mediated signal pathway via the production of IL-10 [71]. 
A certain double-stranded RNA virus, a ligand of TLR3, triggered the expression of IL-10 through IRF3 signaling [72] and that activation of TLR3 signaling induced IRF3 activation [73]. TLR3 and IL-10 participate in the suppression or killing of activated HSCs and Kupffer cells in a variety of models of liver injury. Recent investigations suggest that TRIF-regulated IRF3 binds to the promoter region of the TNF- $\alpha$ gene and upregulates transcription in chronic ethanol-exposed macrophages contributing to alcoholinduced steatosis [74]. These findings suggest that TLRs play a "gate keeper" role in ALD.

\section{Chemokines}

Members of the CXC family of chemokines include IL-8 and growth-regulated $\alpha$-protein (Gro- $\alpha$ ). These mediators attract polymorphonuclear leukocytes which are the predominant inflammatory cells that infiltrate the livers of patients with ALD. In patients with AH, expression of these chemokines in the liver correlates with the severity of portal hypertension and patient survival $[75,76]$.

CCL2, referred to as monocyte chemotactic peptide-1 (MCP-1), is a member of the CC chemokine family. Its expression can be induced in many cell types, including inflammatory cells, hepatocytes, and hepatic stellate cells. CCR2 is the only known receptor for CCL2 and is expressed on monocytes, T lymphocytes, and basophils [77]. MCP-1 regulates adhesion molecules and proinflammatory cytokines TNF- $\alpha$, IL-1 $\beta$, and IL-6 [78]. The pivotal role of MCP-1 in ALD was recognized by showing higher amounts of MCP-1 as compared to other CC chemokines, macrophage inflammatory protein $1 \alpha$ (MIP- $1 \alpha)$, and MIP- $1 \beta$, in the liver and mononuclear cells of patients with AH [79]. And MCP-1 is important in the modulation of proinflammatory cytokines [80]. Deficiency of MCP-1 protects mice against ALD, independent of CCR2, by inhibition of proinflamma-tory cytokines and induction of fatty acid oxidation, linking chemokines to hepatic lipid metabolism [81].

\section{Inflammasomes}

The inflammasome is a multiprotein oligomer consisting of caspase-1, PYCARD, and NALP that mediate the response to cellular danger signals activating and recruiting inflammatory cells. Procaspase- 1 is activated by the inflammasome and cleaves pro-IL-1 $\beta$ into the bioactive IL-1 $\beta$ [82]. The inflammasome also promotes the cleavage of pro-IL-18 into IL-18 to induce IFN- $\gamma$ secretion and natural killer cell activation, cleavage and inactivation of IL-33 [83, 84]. Inflammatory stimuli also drive activation of cytosolic caspase activation and recruitment domain (CARD) that recruit ASC and caspase- 1 to assemble into the inflammasome. Inflammasome and IL-1 $\beta$ are activated in ALD patient or rodent animal model [85]. Recent studies demonstrated mRNA expression of several inflammasomes in the liver thus suggesting that inflammasome activation is a component of the liver pathophysiology in ALD [86].

\section{Oxidative Stress}

Oxidative stress is caused by excess ROS production, which leads to apoptosis and necrosis. ROS can also lead to a free radical chain reaction with unsaturated fatty acids generating toxic lipid intermediates. Oxidant stress is a pathogenic factor for the onset of ALD and nonalcoholic fatty liver disease (NAFLD). In vivo models of alcohol infusion induce lipid peroxidation because of increased free radical formation and decreased hepatic antioxidants, such as glutathione (GSH) [87]. In addition to GSH, other liver antioxidants, such as vitamin $\mathrm{A}$, vitamin $\mathrm{C}$, and bilirubin, and enzymes, such as superoxide dismutase and catalase, remove ROS. Moreover, treatment with an inhibitor of alcohol oxidation, such as 4-methylpyrazole, or an antioxidant, such as trolox, effectively prevented or reduced alcohol-induced toxicity, thereby demonstrating the importance of oxidant stress in the pathogenesis of ALD [88]. The catalytic activity of the cytochrome $\mathrm{P} 450$ enzymes requires oxygen activation, which results in the generation of ROS, such as the superoxide anion $\left(\mathrm{O}_{2}{ }^{-}\right)$, hydrogen peroxide $\left(\mathrm{H}_{2} \mathrm{O}_{2}\right)$, and the hydroxyl radical $(\cdot \mathrm{OH})$. Activated Kupffer cells are responsible for the release of various mediators, such as proinflammatory cytokines including TNF- $\alpha$, IL-1, and ROS. ROS participates in inflammation and modulation of hepatocyte metabolism [89]. There is increased production of ROS in ALD. ROS can be released from a variety of sources, such as activated Kupffer cells, CYP2E1, and NADPH oxidase. NADPH oxidase can enhance the activation of NF- $\kappa \mathrm{B}$ and phosphorylate the ERK1/2 and p38 MAPK kinases that amplify Kupffer cell production of TNF- $\alpha$ [90]. Thus, ROS is highly involved in ALD.

\section{Microflora Products}

The importance of bacterial translocation in the pathogenesis of ALD has been demonstrated in many reports. Intestinal tight junction dysfunction [91-94] or bacterial proliferation $[95,96]$ caused by alcohol or its metabolites such as acetaldehyde enhance bacterial translocation into the liver, which induces the activation of Kupffer cells to release various proinflammatory cytokines and chemokines [97, 98] and increase acute LPS translocation. Chronic alcohol feeding causes structural changes in the gastrointestinal tract that might contribute to LPS translocation [99]. Continuous intragastric feeding of alcohol results in intestinal bacterial overgrowth and enteric dysbiosis, which is due to alcoholinduced downregulation of expression of several intestinal antimicrobial molecules [100]. In the intestine, disruption of tight junctions may lead to increased permeability to pathogens, which appears to be a common mechanism involved in the pathogenesis of ALD. Zonula occludens 1 (ZO-1), occludin, and claudin-5 are the transmembrane proteins that are expressed at the tight junction. Alcohol-treated mice showed loss of ZO-1, occludin, and claudin-5 expression in the colon. Gut-derived endotoxins play a crucial role in liver inflammation. Indeed, gastrointestinal permeability is higher in alcoholics than in normal [92, 101]. Alcoholinduced leaky gut results in the translocation of gramnegative bacteria from the intestinal lumen into the portal 
blood, elevating lipopolysaccharide (LPS) levels and triggering significant inflammation and liver injury [102-104]. The gut-liver axis is associated with alcohol-induced liver injury, both in experimental animal models and in patients of ALD. Intestinal sterilization with antibiotics prevents alcoholinduced liver injury [105]. Probiotic therapy reduces circulating endotoxin derived from intestinal gram-negative bacteria in ALD. These findings indicate that there are close interactions between the liver and intestinal bacteria in ALD.

\section{Treatment}

Abstinence from alcohol is the essential treatment for ALD $[106,107]$, but many ALD patients have difficulty remaining abstinent. Thus, there is an urgent need to develop novel therapeutic interventions. In the experimental setting, there are many animal models of ALD [108-111], but these have some advantages and disadvantages. In the clinical setting, alcoholics with acute hepatitis or cirrhosis have identifiable symptoms and receive treatment. Severe AH patients have a high mortality rate of about $50 \%$, and those who survive have a $70 \%$ probability of developing liver cirrhosis. Nutritional supplementation is necessary for $\mathrm{AH}$ patients because of the prevalence of malnutrition. Only two pharmacological agents, which are corticosteroids and pentoxifylline, are recommended for treating $\mathrm{AH}$. Both are aimed at reducing inflammatory conditions. Corticosteroids reduce cytokine production through transcriptional regulation. On the other hand, pentoxifylline achieves a similar effect through the inhibition of phosphodiesterase. These two agents have relatively strong side effects; hence, they are mainly used for cases of severe AH. New agents are showing promise for treating $\mathrm{AH}$ patients. Many antioxidants are effective in treating alcohol-fed animals. The consequent depletion of antioxidants leads to elevated oxidative stress that contributes to both the genesis and progression of ALD in animal models. However, the effectiveness of antioxidant therapy in human patients with ALD remains obscure. TLR3 activation might be a novel therapeutic strategy for the treatment of ALD. Probiotics are also effective in ALD. Lactobacillus reduces endotoxemia and improves liver injury in the rat model of ALD [112]. Moreover, some clinical studies have indicated the effectiveness of probiotics treatment. However, despite these improvements, an effective treatment for ALD has not yet been established. New treatment methods are required for ALD in the near future.

\section{Conclusion}

Alcohol is one of the most common causes of chronic liver disease in the world [113]. There are numerous factors, such as inflammation, oxidative stress, innate immunity, or fibrosis, that result in the development and progression of ALD. The inflammatory cytokines or chemokines appear to play an important role in ALD. The progression of alcohol-induced liver injury involves some immune cells and hepatocytes through the release of cytokines, chemokines, and inflammasomes. Kupffer cells play an important role in the early stage of ALD, producing TNF- $\alpha$ through TLR4. Based on the understanding of the pathogenesis of ALD, TNF- $\alpha$ is a key to developing new approaches to treatment, which has advanced very little since the introduction of corticosteroid therapy. The development of targeted therapies for ALD is hampered by poor knowledge of the molecular mechanisms involved in its development, particularly in humans, and by the perception that it is an addictive and a self-inflicting disease. We consider that more studies are needed to increase the understanding of the pathogenesis of inflammatory cytokines in order to open new therapeutic avenues for ALD.

\section{References}

[1] J. D. Browning and J. D. Horton, "Molecular mediators of hepatic steatosis and liver injury," Journal of Clinical Investigation, vol. 114, no. 2, pp. 147-152, 2004.

[2] R. N. M. MacSween and A. D. Burt, "Histologic spectrum of alcoholic liver disease," Seminars in Liver Disease, vol. 6, no. 3, pp. 221-232, 1986.

[3] P. S. Haber, R. Warner, D. Seth, M. D. Gorrell, and G. W. McCaughan, "Pathogenesis and management of alcoholic hepatitis," Journal of Gastroenterology and Hepatology, vol. 18, no. 12, pp. 1332-1344, 2003.

[4] T. Tsujimoto, S. Kuriyama, M. Yamazaki et al., "Augmented hepatocellular carcinoma progression and depressed Kupffer cell activity in rat cirrhotic livers," International Journal of Oncology, vol. 18, no. 1, pp. 41-47, 2001.

[5] T. Kitazawa, Y. Nakatani, M. Fujimoto, N. Tamura, M. Uemura, and H. Fukui, "The production of tumor necrosis factor- $\alpha$ by macrophages in rats with acute alcohol loading," Alcoholism, vol. 27, no. 8, pp. 72S-75S, 2003.

[6] Y. Iimuro, R. M. Gallucci, M. Luster, H. Kono, and R. G. Thurman, "Antibodies to tumor necrosis factor alfa attenuate hepatic necrosis and inflammation caused by chronic exposure to ethanol in the rat," Hepatology, vol. 26, no. 6, pp. 1530-1537, 1997.

[7] R. K. Rao, A. Seth, and P. Sheth, "Recent advances in alcoholic liver disease. I. Role of intestinal permeability and endotoxemia in alcoholic liver disease," The American Journal of Physiology: Gastrointestinal and Liver Physiology, vol. 286, no. 6, pp. G881G884, 2004.

[8] P.-H. Park, V. Thakur, M. T. Pritchard, M. R. McMullen, and L. E. Nagy, "Regulation of Kupffer cell activity during chronic ethanol exposure: role of adiponectin," Journal of Gastroenterology and Hepatology, vol. 21, no. 3, pp. S30-S33, 2006.

[9] A. Canbay, A. E. Feldstein, H. Higuchi et al., "Kupffer cell engulfment of apoptotic bodies stimulates death ligand and cytokine expression," Hepatology, vol. 38, no. 5, pp. 1188-1198, 2003.

[10] C. J. McClain, Z. Song, S. S. Barve, D. B. Hill, and I. Deaciuc, "Recent advances in alcoholic liver disease. IV. Dysregulated cytokine metabolism in alcoholic liver disease," The American Journal of Physiology: Gastrointestinal and Liver Physiology, vol. 287, no. 3, pp. G497-G502, 2004.

[11] H. Tilg and A. M. Diehl, "Cytokines in alcoholic and nonalcoholic steatohepatitis," The New England Journal of Medicine, vol. 343, no. 20, pp. 1467-1467, 2000.

[12] B. Gao, "Cytokines, STATs and liver disease," Cellular \& Molecular Immunology, vol. 2, no. 2, pp. 92-100, 2005. 
[13] F. Garcin, G. Lau You Hin, and J. Cote, "Aldehyde dehydrogenase in drosophila: developmental and functional aspects," Alcohol, vol. 2, no. 1, pp. 85-89, 1985.

[14] C. S. Lieber and L. M. DeCarli, "The role of the hepatic microsomal ethanol oxidizing system (MEOS) for ethanol metabolism in vivo," Journal of Pharmacology and Experimental Therapeutics, vol. 181, no. 2, pp. 279-287, 1972.

[15] E. P. A. Neve and M. Ingelman-Sundberg, "Molecular basis for the transport of cytochrome P450 2E1 to the plasma membrane," Journal of Biological Chemistry, vol. 275, no. 22, pp. 17130-17135, 2000.

[16] C. S. Lieber, "Metabolic consequences of ethanol," The Endocrinologist, vol. 4, no. 2, pp. 127-139, 1994.

[17] M. Bilzer, F. Roggel, and A. L. Gerbes, "Role of Kupffer cells in host defense and liver disease," Liver International, vol. 26, no. 10, pp. 1175-1186, 2006.

[18] A. M. Diehl, "Recent events in alcoholic liver disease: V. Effects of ethanol on liver regeneration," The American Journal of Physiology: Gastrointestinal and Liver Physiology, vol. 288, no. 1, pp. G1-G6, 2005.

[19] J. Hansen, D. L. Cherwitz, and J. I. Allen, "The role of tumor necrosis factor- $\alpha$ in acute endotoxin-induced hepatotoxicity in ethanol-fed rats," Hepatology, vol. 20, no. 2, pp. 461-474, 1994.

[20] A. Aldred and L. E. Nagy, "Ethanol dissociates hormonestimulated cAMP production from inhibition of TNF- $\alpha$ production in rat Kupffer cells," The American Journal of Physiology: Gastrointestinal and Liver Physiology, vol. 276, no. 1, pp. G98G106, 1999.

[21] D. B. Hill, L. Marsano, D. Cohen, J. Allen, S. Shedlofsky, and C. J. McClain, "Increased plasma interleukin-6 concentrations in alcoholic hepatitis," The Journal of Laboratory and Clinical Medicine, vol. 119, no. 5, pp. 547-552, 1992.

[22] H. Kawaratani, T. Tsujimoto, T. Kitazawa et al., "Innate immune reactivity of the liver in rats fed a choline-deficient L-aminoacid-defined diet," World Journal of Gastroenterology, vol. 14, no. 43, pp. 6655-6661, 2008.

[23] J. Crespo, A. Cayoen, P. Fernendez-Gil et al., "Gene expression of tumor necrosis factor $\alpha$ and TNF-receptors, p55 and p75, in nonalcoholic steatohepatitis patients," Hepatology, vol. 34, no. 6, pp. 1158-1163, 2001.

[24] T. Matsuyama, M. Uemura, M. Ishikawa et al., "Increased von Willebrand factor over decreased ADAMTS13 activity may contribute to the development of liver disturbance and multiorgan failure in patients with alcoholic hepatitis," Alcoholism, vol. 31, no. 1, pp. S27-S35, 2007.

[25] M. Ishikawa, M. Uemura, T. Matsuyama et al., "Potential role of enhanced cytokinemia and plasma inhibitor on the decreased activity of plasma ADAMTS13 in patients with alcoholic hepatitis: relationship to endotoxemia," Alcoholism, vol. 34, no. 1, pp. S25-S33, 2010.

[26] M. Uemura, Y. Fujimura, T. Matsuyama et al., "Potential role of ADAMTS13 in the progression of alcoholic hepatitis," Current Drug Abuse Reviews, vol. 1, no. 2, pp. 188-196, 2008.

[27] E. Akriviadis, R. Botla, W. Briggs, S. Han, T. Reynolds, and O. Shakil, "Pentoxifylline improves short-term survival in severe acute alcoholic hepatitis: a double-blind, placebo-controlled trial," Gastroenterology, vol. 119, no. 6, pp. 1637-1648, 2000.

[28] H. Tilg, R. Jalan, A. Kaser et al., "Anti-tumor necrosis factoralpha monoclonal antibody therapy in severe alcoholic hepatitis," Journal of Hepatology, vol. 38, no. 4, pp. 419-425, 2003.
[29] H. Kawaratani, T. Tsujimoto, T. Kitazawa, H. Yoshiji, M. Uemura, and H. Fukui, "Therapeutic effects of cytokine modulator Y-40138 in the rat alcoholic liver disease model," Journal of Gastroenterology and Hepatology, vol. 26, no. 4, pp. 775-783, 2011.

[30] N. Sheron, G. Bird, J. Goka, G. Alexander, and R. Williams, "Elevated plasma interleukin- 6 and increased severity and mortality in alcoholic hepatitis," Clinical and Experimental Immunology, vol. 84, no. 3, pp. 449-453, 1991.

[31] F. Hong, W.-H. Kim, Z. Tian et al., "Elevated interleukin-6 during ethanol consumption acts as a potential endogenous protective cytokine against ethanol-induced apoptosis in the liver: involvement of induction of Bcl-2 and Bcl-xL proteins," Oncogene, vol. 21, no. 1, pp. 32-43, 2002.

[32] J. Latvala, J. Hietala, H. Koivisto, K. Järvi, P. Anttila, and O. Niemelä, "Immune responses to ethanol metabolites and cytokine profiles differentiate alcoholics with or without liver disease," American Journal of Gastroenterology, vol. 100, no. 6, pp. 1303-1310, 2005.

[33] O. El-Assal, F. Hong, W.-H. Kim, S. Radaeva, and B. Gao, "IL-6-deficient mice are susceptible to ethanol-induced hepatic steatosis: IL-6 protects against ethanol-induced oxidative stress and mitochondrial permeability transition in the liver," Cellular \& molecular immunology, vol. 1, no. 3, pp. 205-211, 2004.

[34] X. Zhang, S. Tachibana, H. Wang et al., "Interleukin-6 is an important mediator for mitochondrial DNA repair after alcoholic liver injury in mice," Hepatology, vol. 52, no. 6, pp. 21372147, 2010.

[35] B. Rabe, A. Chalaris, U. May et al., "Transgenic blockade of interleukin 6 transsignaling abrogates inflammation," Blood, vol. 111, no. 3, pp. 1021-1028, 2008.

[36] P. Mathurin, Q.-G. Deng, A. Keshavarzian, S. Choudhary, E. W. Holmes, and H. Tsukamoto, "Exacerbation of alcoholic liver injury by enteral endotoxin in rats," Hepatology, vol. 32, no. 5, pp. 1008-1017, 2000.

[37] C. M. Hawrylowicz and A. O'Garra, "Potential role of interleukin-10-secreting regulatory $\mathrm{T}$ cells in allergy and asthma," Nature Reviews Immunology, vol. 5, no. 4, pp. 271-283, 2005.

[38] H. Louis, O. Le Moine, M. Goldman, and J. Devière, "Modulation of liver injury by interleukin-10," Acta Gastro-Enterologica Belgica, vol. 66, no. 1, pp. 7-14, 2003.

[39] D. F. Fiorentino, A. Zlotnik, P. Vieira et al., "IL-10 acts on the antigen-presenting cell to inhibit cytokine production by Th1 cells," Journal of Immunology, vol. 146, no. 10, pp. 3444-3451, 1991.

[40] R. De Waal Malefyt, J. Abrams, B. Bennett, C. G. Figdor, and J. E. De Vries, "Interleukin 10(IL-10) inhibits cytokine synthesis by human monocytes: an autoregulatory role of IL-10 produced by monocytes," Journal of Experimental Medicine, vol. 174, no. 5, pp. 1209-1220, 1991.

[41] D. B. Hill, N. B. D’Souza, E. Y. Lee, R. Burikhanov, I. V. Deaciuc, and W. J. S. De Villiers, "A role for interleukin-10 in alcoholinduced liver sensitization to bacterial lipopolysaccharide," Alcoholism, vol. 26, no. 1, pp. 74-82, 2002.

[42] A. M. Miller, H. Wang, A. Bertola et al., "Inflammationassociated interleukin-6/signal transducer and activator of transcription 3 activation ameliorates alcoholic and nonalcoholic fatty liver diseases in interleukin-10-deficient mice," Hepatology, vol. 54, no. 3, pp. 846-856, 2011.

[43] A. A. Nanji, K. Jokelainen, A. Rahemtulla et al., "Activation of nuclear factor kappa B and cytokine imbalance in experimental 
alcoholic liver disease in the rat," Hepatology, vol. 30, no. 4, pp. 934-943, 1999.

[44] J. Jura, P. Wegrzyn, M. Korostyński et al., "Identification of interleukin-1 and interleukin-6-responsive genes in human monocyte-derived macrophages using microarrays," Biochimica et Biophysica Acta, vol. 1779, no. 6-7, pp. 383-389, 2008.

[45] E. V. Acosta-Rodriguez, G. Napolitani, A. Lanzavecchia, and F. Sallusto, "Interleukins $1 \beta$ and 6 but not transforming growth factor- $\beta$ are essential for the differentiation of interleukin 17producing human T helper cells," Nature Immunology, vol. 8, no. 9, pp. 942-949, 2007.

[46] C. T. Weaver, R. D. Hatton, P. R. Mangan, and L. E. Harrington, "IL-17 family cytokines and the expanding diversity of effector T cell lineages," Annual Review of Immunology, vol. 25, pp. 821852, 2007.

[47] F. Lafdil, A. M. Miller, S. H. Ki, and B. Gao, "Th17 cells and their associated cytokines in liver diseases," Cellular and Molecular Immunology, vol. 7, no. 4, pp. 250-254, 2010.

[48] A. Lemmers, C. Moreno, T. Gustot et al., "The interleukin-17 pathway is involved in human alcoholic liver disease," Hepatology, vol. 49, no. 2, pp. 646-657, 2009.

[49] A. A. Nanji, S. Zhao, S. M. H. Sadrzadeh, A. J. Dannenberg, S. R. Tahan, and D. J. Waxman, "Markedly enhanced cytochrome P450 2E1 induction and lipid peroxidation is associated with severe liver injury in fish oil-ethanol-fed rats," Alcoholism, vol. 18, no. 5, pp. 1280-1285, 1994.

[50] S. H. Ki, O. Park, M. Zheng et al., "Interleukin-22 treatment ameliorates alcoholic liver injury in a murine model of chronicbinge ethanol feeding: role of signal transducer and activator of transcription 3," Hepatology, vol. 52, no. 4, pp. 1291-1300, 2010.

[51] C. A. Dinarello, "Interleukin- $1 \beta$ and the autoinflammatory diseases," The New England Journal of Medicine, vol. 360, no. 23, pp. 2467-2470, 2009.

[52] J. Petrasek, S. Bala, T. Csak et al., "IL-1 receptor antagonist ameliorates inflammasome-dependent alcoholic steatohepatitis in mice," The Journal of Clinical Investigation, vol. 122, pp. 34763489, 2012.

[53] H. Tilg, A. Wilmer, W. Vogel et al., "Serum levels of cytokines in chronic liver diseases," Gastroenterology, vol. 103, no. 1, pp. 264274, 1992.

[54] M. Mertens and J. A. Singh, "Anakinra for rheumatoid arthritis," Cochrane Database of Systematic Reviews, no. 1, Article ID CD005121, 2009.

[55] S. Akira, S. Uematsu, and O. Takeuchi, "Pathogen recognition and innate immunity," Cell, vol. 124, no. 4, pp. 783-801, 2006.

[56] T. Gustot, A. Lemmers, C. Moreno et al., "Differential liver sensitization to toll-like receptor pathways in mice with alcoholic fatty liver," Hepatology, vol. 43, no. 5, pp. 989-1000, 2006.

[57] I. Hritz, P. Mandrekar, A. Velayudham et al., "The critical role of toll-like receptor (TLR) 4 in alcoholic liver disease is independent of the common TLR adapter MyD88," Hepatology, vol. 48, no. 4, pp. 1224-1231, 2008.

[58] E. Seki and D. A. Brenner, "Toll-like receptors and adaptor molecules in liver disease: update," Hepatology, vol. 48, no. 1, pp. 322-335, 2008.

[59] M. Isogawa, M. D. Robek, Y. Furuichi, and F. V. Chisari, “Tolllike receptor signaling inhibits hepatitis B virus replication in vivo," Journal of Virology, vol. 79, no. 11, pp. 7269-7272, 2005.

[60] R. Medzhitov, P. Preston-Hurlburt, and C. A. Janeway Jr., "A human homologue of the Drosophila toll protein signals activation of adaptive immunity," Nature, vol. 388, no. 6640, pp. 394397, 1997.
[61] M. Yamamoto, S. Sato, H. Hemmi et al., "Role of adaptor TRIF in the MyD88-independent toll-like receptor signaling pathway," Science, vol. 301, no. 5633, pp. 640-643, 2003.

[62] G. Szabo, J. Petrasek, and S. Bala, "Innate immunity and alcoholic liver disease," Digestive Diseases, vol. 30, pp. S55-S60, 2012.

[63] G. Szabo, P. Mandrekar, J. Petrasek, and D. Catalano, "The unfolding web of innate immune dysregulation in alcoholic liver injury," Alcoholism, vol. 35, no. 5, pp. 782-786, 2011.

[64] M. Yin, B. U. Bradford, M. D. Wheeler et al., "Reduced early alcohol-induced liver injury in CD14-deficient mice," Journal of Immunology, vol. 166, no. 7, pp. 4737-4742, 2001.

[65] Y.-H. Paik, R. F. Schwabe, R. Bataller, M. P. Russo, C. Jobin, and D. A. Brenner, "Toll-like receptor 4 mediates inflammatory signaling by bacterial lipopolysaccharide in human hepatic stellate cells," Hepatology, vol. 37, no. 5, pp. 1043-1055, 2003.

[66] L. E. Nagy, "Recent insights into the role of the innate immune system in the development of alcoholic liver disease," Experimental Biology and Medicine, vol. 228, no. 8, pp. 882-890, 2003.

[67] M. Fujimoto, M. Uemura, Y. Nakatani et al., "Plasma endotoxin and serum cytokine levels in patients with alcoholic hepatitis: relation to severity of liver disturbance," Alcoholism, vol. 24, no. 4, pp. S48-S54, 2000.

[68] H. Fukui, B. Brauner, J. C. Bode, and C. Bode, "Plasma endotoxin concentrations in patients with alcoholic and non-alcoholic liver disease: reevaluation with an improved chromogenic assay," Journal of Hepatology, vol. 12, no. 2, pp. 162-169, 1991.

[69] T. Gustot, A. Lemmers, C. Moreno et al., "Differential liver sensitization to toll-like receptor pathways in mice with alcoholic fatty liver," Hepatology, vol. 43, no. 5, pp. 989-1000, 2006.

[70] P. Mandrekar, D. Catalano, B. White, and G. Szabo, "Moderate alcohol intake in humans attenuates monocyte inflammatory responses: inhibition of nuclear regulatory factor kappa B and induction of interleukin 10," Alcoholism, vol. 30, no. 1, pp. 135139, 2006.

[71] J. S. Byun, Y. G. Suh, H. S. Yi et al., "Activation of toll-like receptor 3 attenuates alcoholic liver injury by stimulating Kupffer cells and stellate cells to produce interleukin-10 in mice," Journal of Hepatology, vol. 58, pp. 342-349, 2013.

[72] M. Samanta, D. Iwakiri, and K. Takada, "Epstein-Barr virusencoded small RNA induces IL-10 through RIG-I-mediated IRF-3 signaling," Oncogene, vol. 27, no. 30, pp. 4150-4160, 2008.

[73] E. Meylan and J. Tschopp, "Toll-like receptors and RNA helicases: two parallel ways to trigger antiviral responses," Molecular Cell, vol. 22, no. 5, pp. 561-569, 2006.

[74] X.-J. Zhao, Q. Dong, J. Bindas et al., "TRIF and IRF-3 binding to the TNF promoter results in macrophage TNF dysregulation and steatosis induced by chronic ethanol," Journal of Immunology, vol. 181, no. 5, pp. 3049-3056, 2008.

[75] M. Dominguez, R. Miquel, J. Colmenero et al., "Hepatic expression of CXC chemokines predicts portal hypertension and survival in patients with alcoholic hepatitis," Gastroenterology, vol. 136, no. 5, pp. 1639-1650, 2009.

[76] J. Colmenero, R. Bataller, P. Sancho-Bru et al., "Hepatic expression of candidate genes in patients with alcoholic hepatitis: correlation with disease severity," Gastroenterology, vol. 132, no. 2, pp. 687-697, 2007.

[77] K. J. Simpson, N. C. Henderson, C. L. Bone-Larson, N. W. Lukacs, C. M. Hogaboam, and S. L. Kunkel, "Chemokines in the pathogenesis of liver disease: so many players with poorly defined roles," Clinical Science, vol. 104, no. 1, pp. 47-63, 2003. 
[78] Y. Jiang, D. I. Beller, G. Frendl, and D. T. Graves, "Monocyte chemoattractant protein-1 regulates adhesion molecule expression and cytokine production in human monocytes," Journal of Immunology, vol. 148, no. 8, pp. 2423-2428, 1992.

[79] N. C. Fisher, D. A. H. Neil, A. Williams, and D. H. Adams, "Serum concentrations and peripheral secretion of the beta chemokines monocyte chemoattractant protein 1 and macrophage inflammatory protein $1 \alpha$ in alcoholic liver disease," Gut, vol. 45, no. 3, pp. 416-420, 1999.

[80] B. Lu, B. J. Rutledge, L. Gu et al., "Abnormalities in monocyte recruitment and cytokine expression in monocyte chemoattractant protein 1-deficient mice," The Journal of Experimental Medicine, vol. 187, no. 4, pp. 601-608, 1998.

[81] P. Mandrekar, A. Ambade, A. Lim, G. Szabo, and D. Catalano, "An essential role for monocyte chemoattractant protein-1 in alcoholic liver injury: regulation of proinflammatory cytokines and hepatic steatosis in mice," Hepatology, vol. 54, no. 6, pp. 2185-2197, 2011.

[82] K. Schroder and J. Tschopp, “The inflammasomes," Cell, vol. 140, no. 6, pp. 821-832, 2010.

[83] C. Cayrol and J.-P. Girard, "The IL-1-like cytokine IL-33 is inactivated after maturation by caspase-1," Proceedings of the National Academy of Sciences of the United States of America, vol. 106, no. 22, pp. 9021-9026, 2009.

[84] F. Martinon, A. Mayor, and J. Tschopp, "The inflammasomes: guardians of the body," Annual Review of Immunology, vol. 27, pp. 229-265, 2009.

[85] S. L. Valles, A. M. Blanco, I. Azorin et al., "Chronic ethanol consumption enhances interleukin-1-mediated signal transduction in rat liver and in cultured hepatocytes," Alcoholism, vol. 27, no. 12, pp. 1979-1986, 2003.

[86] M. Lech, A. Avila-Ferrufino, V. Skuginna, H. E. Susanti, and H.J. Anders, "Quantitative expression of RIG-like helicase, NODlike receptor and inflammasome-related mRNAs in humans and mice," International Immunology, vol. 22, no. 9, pp. 717-728, 2010.

[87] Y. Iimuro, B. U. Bradford, S. Yamashina et al., "The glutathione precursor L-2-oxothiazolidine-4-carboxylic acid protects against liver injury due to chronic enteral ethanol exposure in the rat," Hepatology, vol. 31, no. 2, pp. 391-398, 2000.

[88] D. Wu and A. I. Cederbaum, "Ethanol-induced apoptosis to stable HepG2 cell lines expressing human cytochrome P-4502E1," Alcoholism, vol. 23, no. 1, pp. 67-76, 1999.

[89] M. D. Wheeler, "Endotoxin and kupffer cell activation in alcoholic liver disease," Alcohol Research and Health, vol. 27, no. 4, pp. 300-306, 2003.

[90] E. Albano, "Oxidative mechanisms in the pathogenesis of alcoholic liver disease," Molecular Aspects of Medicine, vol. 29, no. 12, pp. 9-16, 2008.

[91] A. Keshavarzian, A. Farhadi, C. B. Forsyth et al., "Evidence that chronic alcohol exposure promotes intestinal oxidative stress, intestinal hyperpermeability and endotoxemia prior to development of alcoholic steatohepatitis in rats," Journal of Hepatology, vol. 50, no. 3, pp. 538-547, 2009.

[92] A. Keshavarzian, E. W. Holmes, M. Patel, F. Iber, J. Z. Fields, and S. Pethkar, "Leaky gut in alcoholic cirrhosis: a possible mechanism for alcohol- induced liver damage," American Journal of Gastroenterology, vol. 94, no. 1, pp. 200-207, 1999.

[93] S. Bala, M. Marcos, K. Kodys et al., "Up-regulation of microRNA-155 in macrophages contributes to increased Tumor Necrosis Factor $\alpha(\mathrm{TNF} \alpha)$ production via increased mRNA half-life in alcoholic liver disease," Journal of Biological Chemistry, vol. 286, no. 2, pp. 1436-1444, 2011.

[94] P. Sheth, A. Seth, K. J. Atkinson et al., "Acetaldehyde dissociates the PTP1B-E-cadherin- $\beta$-catenin complex in Caco- 2 cell monolayers by a phosphorylation-dependent mechanism," The Biochemical Journal, vol. 402, no. 2, pp. 291-300, 2007.

[95] Z. Yumuk, S. Ozdemirci, B. Faruk Erden, and V. Dundar, "The effect of long-term ethanol feeding on Brucella melitensis infection of rats," Alcohol and Alcoholism, vol. 36, no. 4, pp. 314317, 2001.

[96] M. J. Kavanaugh, C. Clark, M. Goto et al., "Effect of acute alcohol ingestion prior to burn injury on intestinal bacterial growth and barrier function," Burns, vol. 31, no. 3, pp. 290-296, 2005.

[97] M. D. Wheeler, H. Kono, M. Yin et al., “The role of kupffer cell oxidant production in early ethanol-induced liver disease," Free Radical Biology and Medicine, vol. 31, no. 12, pp. 1544-1549, 2001.

[98] V. Thakur, M. R. McMullen, M. T. Pritchard, and L. E. Nagy, "Regulation of macrophage activation in alcoholic liver disease," Journal of Gastroenterology and Hepatology, vol. 22, no. 1, pp. S53-S56, 2007.

[99] R. Rao, "Endotoxemia and gut barrier dysfunction in alcoholic liver disease," Hepatology, vol. 50, no. 2, pp. 638-644, 2009.

[100] A. W. Yan, D. E. Fouts, J. Brandl et al., "Enteric dysbiosis associated with a mouse model of alcoholic liver disease," Hepatology, vol. 53, no. 1, pp. 96-105, 2011.

[101] A. Keshavarzian, J. Z. Fields, J. Vaeth, and E. W. Holmes, “The differing effects of acute and chronic alcohol on gastric and intestinal permeability," American Journal of Gastroenterology, vol. 89, no. 12, pp. 2205-2211, 1994.

[102] M. A. Choudhry, N. Fazal, M. Goto, R. L. Gamelli, and M. M. Sayeed, "Gut-associated lymphoid T cell suppression enhances bacterial translocation in alcohol and burn injury," The American Journal of Physiology: Gastrointestinal and Liver Physiology, vol. 282, no. 6, pp. G937-G947, 2002.

[103] A. Keshavarzian, S. Choudhary, E. W. Holmes et al., "Preventing gut leakiness by oats supplementation ameliorates alcoholinduced liver damage in rats," Journal of Pharmacology and Experimental Therapeutics, vol. 299, no. 2, pp. 442-448, 2001.

[104] G. Szabo and S. Bala, "Alcoholic liver disease and the gut-liver axis," World Journal of Gastroenterology, vol. 16, no. 11, pp. 1321$1329,2010$.

[105] Y. Adachi, L. E. Moore, B. U. Bradford, W. Gao, and R. G. Thurman, "Antibiotics prevent liver injury in rats following longterm exposure to ethanol," Gastroenterology, vol. 108, no. 1, pp. 218-224, 1995.

[106] B. Gao and R. Bataller, "Alcoholic liver disease: pathogenesis and new therapeutic targets," Gastroenterology, vol. 141, no. 5, pp. 1572-1585, 2011.

[107] R. S. O'Shea, S. Dasarathy, and A. J. McCullough, "Alcoholic liver disease," Hepatology, vol. 51, pp. 307-328, 2010.

[108] C. S. Lieber and L. M. DeCarli, "Alcoholic liver injury: experimental models in rats and baboons," Advances in Experimental Medicine and Biology, vol. 59, pp. 379-393, 1975.

[109] H. Tsukamoto, S. W. French, and N. Benson, "Severe and progressive steatosis and focal necrosis in rat liver induced by continuous intragastric infusion of ethanol and low fat diet," Hepatology, vol. 5, no. 2, pp. 224-232, 1985.

[110] H. Tsukamoto, S. J. Towner, L. M. Ciofalo, and S. W. French, "Ethanol-induced liver fibrosis in rats fed high fat diet," Hepatology, vol. 6, no. 5, pp. 814-822, 1986. 
[111] S. W. French, "Intragastric ethanol infusion model for cellular and molecular studies of alcoholic liver disease," Journal of Biomedical Science, vol. 8, no. 1, pp. 20-27, 2001.

[112] A. A. Nanji, U. Khettry, and S. M. H. Sadrzadeh, "Lactobacillus feeding reduces endotoxemia and severity of experimental alcoholic liver (disease)," Proceedings of the Society for Experimental Biology and Medicine, vol. 205, no. 3, pp. 243-247, 1994.

[113] T. Tsujimoto, H. Kawaratani, H. Yoshiji, M. Uemura, and H. Fukui, "Recent developments in the treatment of alcoholic chronic pancreatitis," Current Drug Abuse Reviews, vol. 1, no. 2, pp. 197-202, 2008. 


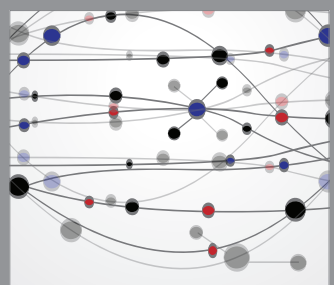

The Scientific World Journal
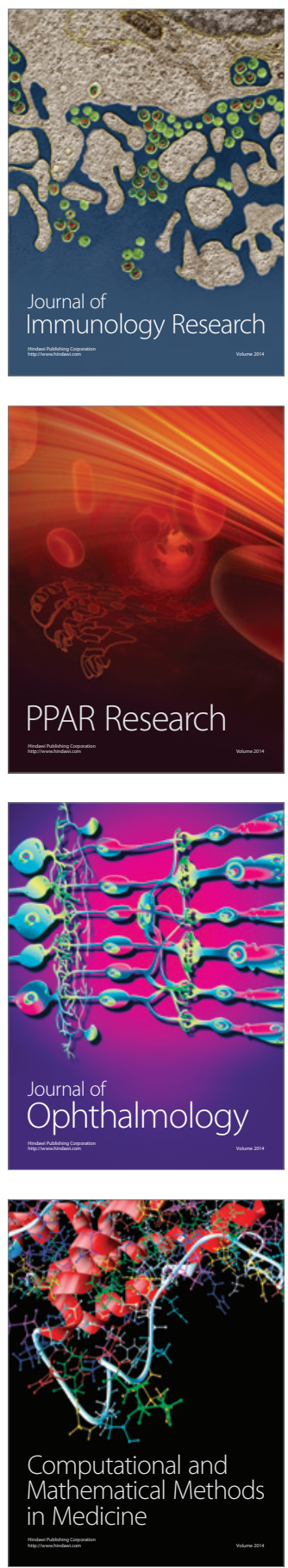

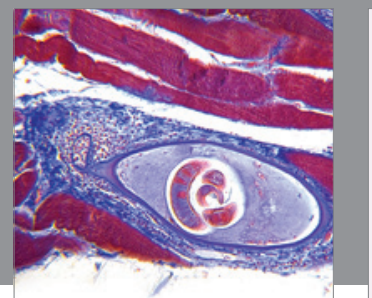

Gastroenterology

Research and Practice
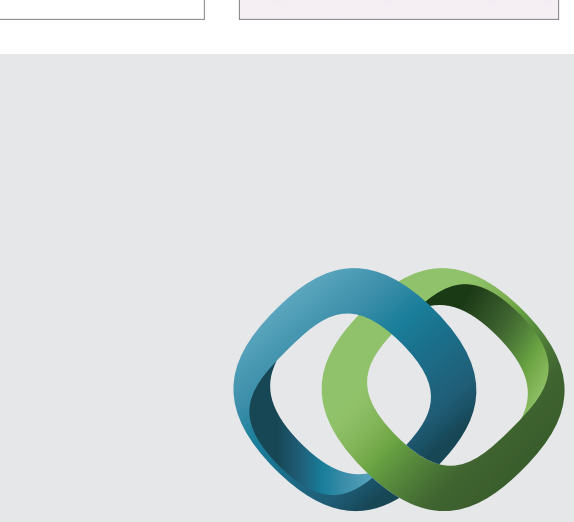

\section{Hindawi}

Submit your manuscripts at

http://www.hindawi.com
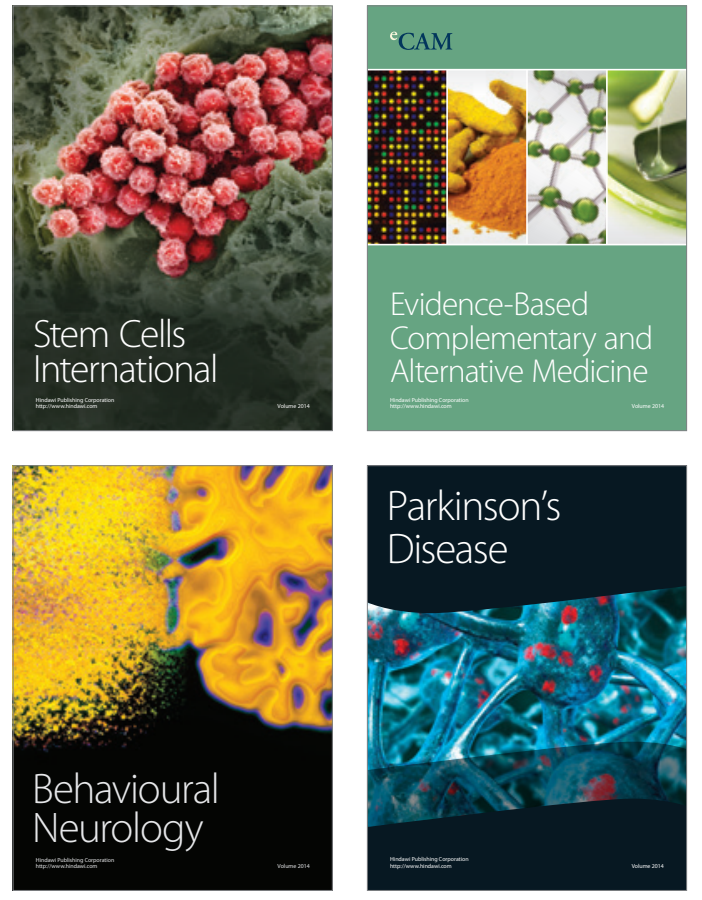
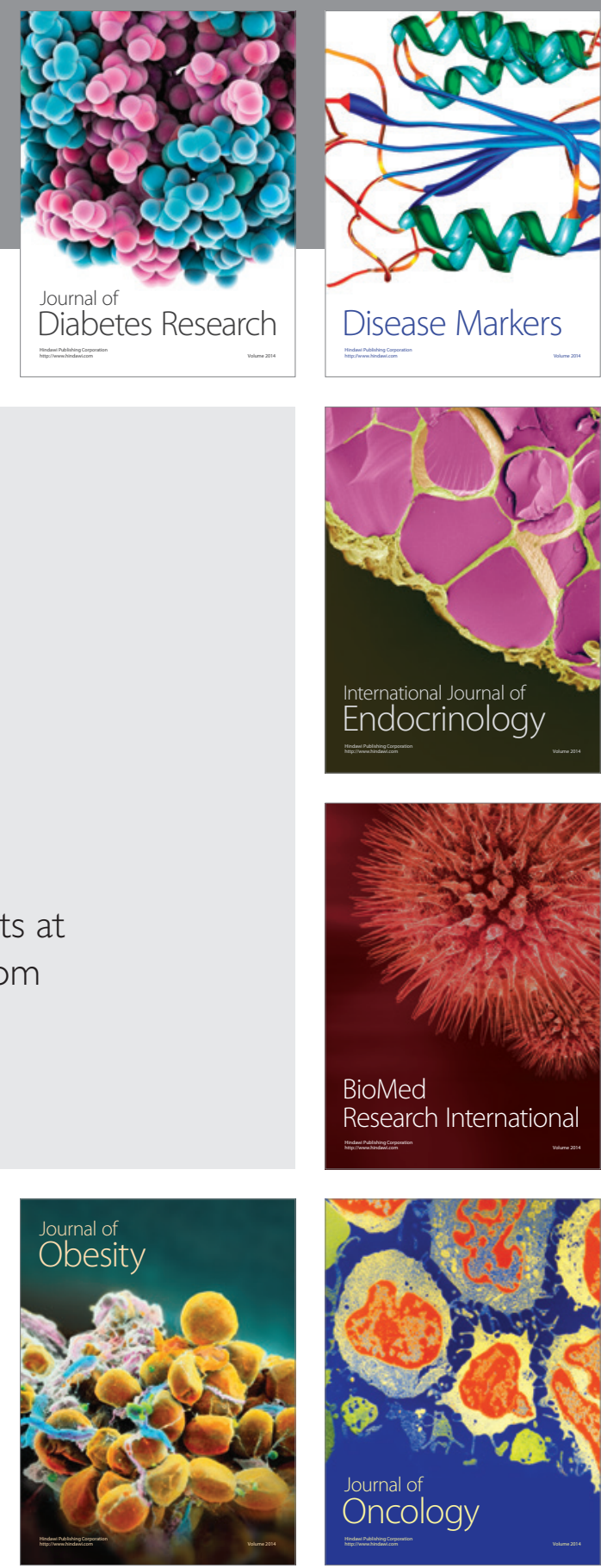

Disease Markers
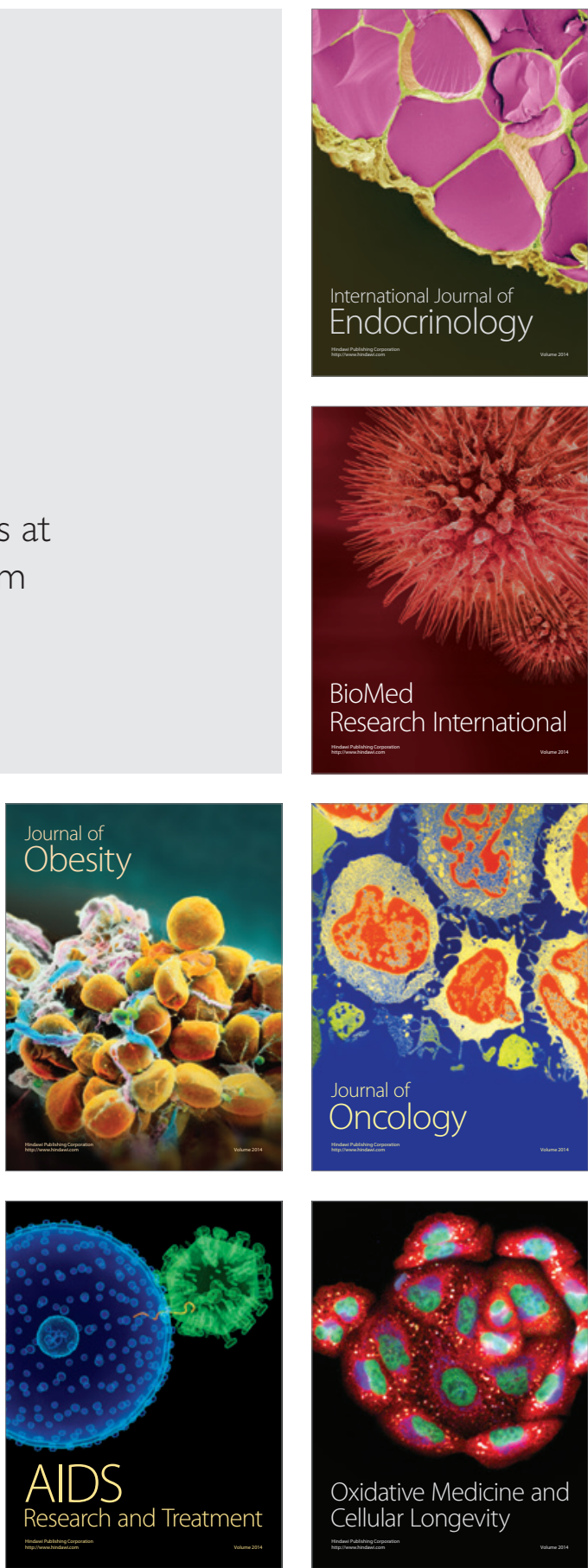